\title{
Re-evaluating Safety and Effectiveness of Dabigatran Versus Warfarin in a Nationwide Data Environment: A Prevalent New-User Design Study
}

\author{
Hui-Min Diana Lin ${ }^{1,2,3}$ (D) Chao-Lun Lai ${ }^{3,4,5} \cdot$ Yaa-Hui Dong ${ }^{6,7} \cdot$ Yu-Kang Tu ${ }^{1,3} \cdot K$. Arnold Chan ${ }^{1,2}$ (1) $\cdot$ Samy Suissa ${ }^{8,9,10}$
}

Published online: 25 June 2019

(c) The Author(s) 2019

\begin{abstract}
Introduction The new user cohort design is widely used to assess the effects of a new drug, such as dabigatran, but inherently excludes some users due to prior use of the comparator drug, for example warfarin. The prevalent new-user design offers a solution that includes all eligible users of the new drug.

Objective To evaluate the safety and effectiveness of dabigatran versus warfarin in non-valvular atrial fibrillation (NVAF) patients with prevalent new-user design.

Methods Taiwan National Health Insurance and mortality data from 2011 through 2015 were utilized. From an incident NVAF cohort, we identified dabigatran initiators as either incident or prevalent (switchers from warfarin) new users. Timeand prescription-based exposure sets were formed for dabigatran initiators to account for prior warfarin prescriptions. A comparable warfarin user was matched on the time-conditional propensity score to the dabigatran initiator in each set. The matched patients were followed for clinical outcomes, with Cox proportional hazards model used to estimate hazard ratios (HRs).

Results There were 10,811 dabigatran initiators, including $22 \%$ prevalent new users (switchers), who formed the exposure sets and were matched 1:1 to warfarin users. Dabigatran use was associated with lower risks of intracranial hemorrhage (HR $0.51 ; 95 \%$ confidence interval [CI] 0.39, 0.66) and gastrointestinal bleeding (HR 0.81; 95\% CI 0.70, 0.92), compared with warfarin use. These effects were similar between the incident and prevalent new users.

Conclusion Using a design that includes both incident and prevalent new users of dabigatran, the use of dabigatran is associated with lower major bleeding risk than warfarin use among patients with incident NVAF.
\end{abstract}

Electronic supplementary material The online version of this article (https://doi.org/10.1007/s40801-019-0156-2) contains supplementary material, which is available to authorized users.

K. Arnold Chan

kachan@ntu.edu.tw

Extended author information available on the last page of the article

\section{Key Points}

The prevalent new user design allows for inclusion of both dabigatran incident new users and prevalent new users in the same analysis where prior use of warfarin was accounted for through the time- and prescriptionbased exposure sets.

Dabigatran use was associated with lower risk of intracranial hemorrhage, gastrointestinal bleeding and allcause mortality compared to warfarin use.

Outcomes among dabigatran incident new users and prevalent new users could be evaluated directly from subgroup analysis, where lower ischemic stroke risk compared to warfarin use was observed among dabigatran incident new users but not prevalent new users. 


\section{Background}

In early post-marketing safety assessment of newly marketed drugs, ideally all users of a new drug should be identified for evaluation of adverse outcomes. Some new drugs are novel therapies for disease conditions that do not have approved drug treatment and thus all users of the new drugs are genuine new users and would not have received any prior treatment. However, for disease conditions with existing drug treatment, newly approved drugs are either initiated in patients after switching from a prior drug (switchers) or initiated for patients without any prior medication for the disease condition (genuine new users). Safety assessment is further complicated by whether the new drug is approved as monotherapy, add-on therapy, or both. The new user design with active comparator has been the most common method in post-marketing safety and effectiveness research to study two drugs among comparable patients [1-4]. Studying exclusively new users of a new drug and new users of the comparator who have not received prior treatment would mimic a clinical trial with sufficient washout duration and to improve internal validity, but would exclude many users of the new drug who are switched from prior drugs in real-world practice. A pertinent example was a study evaluating the risk of angioedema associated with use of drugs targeting the renin-angiotensin-aldosterone system with beta-blocker as the active comparator; the study size was only $30 \%$ of all potential subjects when eligibility was restricted to new users who had no prior use of any study drugs [5]. Studying all eligible users of a new drug would provide more generalizable data and greater statistical power, but the heterogeneity of the new drug users would result in methodology challenges in identifying comparators and making valid inference on comparative safety and effectiveness.

The methodology challenges described above apply to post-marketing safety assessment of non-vitamin $\mathrm{K}$ oral anticoagulant (NOAC) for patients with non-valvular atrial fibrillation (NVAF) [6-12]. After a NOAC was introduced, some patients with prevalent NVAF might have been treated with warfarin suboptimally for years before switching to a NOAC; patients with newly diagnosed NVAF might be initially prescribed warfarin although the NOAC was available and were switched to the NOAC shortly afterwards, and other patients with newly diagnosed atrial fibrillation (AF) would initiate the NOAC without prior use of warfarin [11-15]. Despite the apparent heterogeneity of patients initiating NOAC, safety studies utilizing the active comparator new-user design of NOAC and warfarin have been implemented differently $[6,8,16,17]$. In the early days after approval of NOACs when major bleeding risk was uncertain [18], regulatory agencies understandably would wish to include all NOAC initiators in safety studies rather than only studying the internally valid new users under the conventional research framework. Methodology concerns with including NOAC initiators who switched from warfarin use included under-ascertainment of outcomes of interest during prior warfarin exposure and change in confounder distribution as a result of prior warfarin use [4]. Another concern has been the "depletion of susceptibles" when the prevalent warfarin users who switched to NOAC had a lower bleeding risk $[19,20]$. In prior studies of the newly introduced NOAC dabigatran, the proportion of dabigatran users with prior warfarin exposure ranged from 38 to 51\% [11-15]. Including those who switched from warfarin in the dabigatran new users would result in the heterogeneity described above and excluding these dabigatran users would limit study size and generalizability of the study findings.

Prevalent new user design $[21,22]$ has been proposed recently to account for prior comparator drug use experience and to retain as many users of the newly introduced drug as possible in post-marketing observational studies. In this study design, users of a new drug (some had not used any comparator drug of interest before and others had used a comparator drug for the same indication) are compared against users of a comparator with similar prior exposure experience in the same analysis, accurately reflecting the mix of patients using the new drug in real-world settings. The objective of this study is to implement this novel design to evaluate safety and effectiveness of dabigatran among NVAF patients after its introduction to Taiwan, using warfarin as the comparator and taking into account the methodologic challenges when a substantial portion of dabigatran initiators were switched from warfarin.

\section{Methods}

\subsection{Data Source}

Taiwan National Health Insurance (NHI) is a publiclyfunded single-payer compulsory program started in 1995 with coverage of more than $99 \%$ of the population in Taiwan [23]. A majority of healthcare providers contract with the Taiwan NHI Administration to provide a wide range of medical services. Clinical diagnoses are coded according to the International Classification of Disease Ninth Revision Clinical Modification (ICD-9-CM) through the end of 2015 and transitioned to the Tenth Revision starting in 2016. Diagnoses and drug prescriptions in NHI have been validated particularly in the field of cardiovascular diseases, demonstrating high positive predictive values and consistency for related co-morbidities as well as concomitant medications [24, 25]. For research purposes the NHI claims can be linked to Taiwan National Death Registry 
to provide information on date and cause of death [26]. For this study NHI and Death Registry data from 2011 to 2015 were accessed through the Health and Welfare Data Science Center of the Department of Statistics, Ministry of Health and Welfare in Taiwan.

\subsection{Base Cohort}

We identified patients with incident diagnosis of NVAF (ICD-9-CM 427.3 in ambulatory care or hospital admission claims at any claim position) from 2012 through 2015 with a look-back period of at least 1 year to ascertain prior diagnoses. Patients with mitral stenosis, valvular heart diseases, valve replacement, commissurotomy, heart transplantation, extracorporeal circulation or venous thromboembolism diagnoses 12 months before incident $\mathrm{AF}$ or age under 20 years at the time of first AF diagnosis were excluded. A base cohort was formed with patients having first warfarin or dabigatran prescribed on or after the initial AF diagnosis.

The first warfarin or dabigatran prescribed on or after AF was the cohort entry drug and its prescription date was the cohort entry date. Patients with more than one oral anticoagulant prescribed on cohort entry date were excluded. Patients who received rivaroxaban or apixaban (alternate NOACs) prior to cohort entry date were excluded (Fig. 1). Edoxaban was approved in February 2016 and not reimbursed by NHI until September 2016, and thus is not considered in this study (Table 1).

Dabigatran initiators in the base cohort included both incident and prevalent new users. An incident new user, in some studies referred to as incident user or new user [2], was operationally defined in this study as a new user of dabigatran who did not use oral anticoagulants before (oral anticoagulant naïve), whereas a prevalent new user (switcher) was a dabigatran initiator who had prior use of another oral anticoagulant (oral anticoagulant non-naïve or oral anticoagulant experienced) [12, 13, 27]. In some reports the term initiators is used, which may represent new users of a certain drug regardless of prior use of drugs of the same class [28]. Different terms used in the literature to describe different drug-use scenarios are summarized in Table 2. In this report we use the terms "incident new users" and "new users" interchangeably, as well as "prevalent new users" and "switchers".

Co-morbidities of interest and their corresponding ICD-9-CM codes and NHI specific procedure codes are described in Supplementary Table 1, and concomitant drugs of interest with corresponding Anatomical Therapeutic Chemical Classification System (ATC) codes are described in Supplementary Table 2.

\subsection{Time- and Prescription-based Exposure Sets}

Warfarin and dabigatran prescriptions were individual units to be evaluated to construct exposure sets from the base cohort, while in traditional cohort studies, the unit of interest would be individual patients. An exposure set was formed for every new dabigatran prescription; number of prior warfarin and time since cohort entry to new dabigatran prescription were enumerated among patients initiating dabigatran. Patients who had alternate NOACs prescribed before cohort entry were excluded from the exposure sets. When constructing the exposure sets, we also excluded patients with two oral anticoagulants prescribed on the same day after cohort entry. For each exposure set, there was one dabigatran initial prescription (dabigatran index prescription) and multiple corresponding warfarin prescriptions. Eligible warfarin prescriptions in the exposure set were those who fulfilled the following criteria: (1) time since cohort entry to the warfarin prescription was within 15 days of the interval between cohort entry and dabigatran index prescription, (2) there were same number of prior warfarin prescriptions before the dabigatran index prescription, and (3) they occurred during the same calendar year as the dabigatran index prescription (Fig. 2). Initiating dabigatran prescriptions were then categorized as either an incident new-user exposure set-no warfarin prescription before the initial dabigatran prescription, or a prevalent new user (switcher) exposure set-having at least one warfarin prescription prior to the initial dabigatran prescription. A patient could contribute warfarin prescriptions to multiple exposure sets.

\subsection{Time-conditional Propensity Score (PS)}

Propensity score (PS) estimation with anticoagulant prescription (dabigatran or warfarin) as the dependent binary variable was carried out with conditional logistic regression within each exposure set. Covariates in the regression model included age, gender, co-morbidities, and concomitant medications among the initial dabigatran and warfarin prescriptions at the time of forming or being included in the exposure sets. We ascertained co-morbidities and concomitant medications during the 90 days before the dabigatran or warfarin prescription of interest, as most patients with chronic conditions like NVAF in Taiwan visit physicians every 3 months for chronic medication refills, so diagnoses of both chronic and acute conditions would be captured in the health insurance claims. We estimated PS for every dabigatran and warfarin prescription included in the exposure sets by using the coefficients obtained from the conditional logistic regression model. For new users, PS would be the probability of a patient initiating a dabigatran prescription and among prevalent new users (switchers), PS would be the 


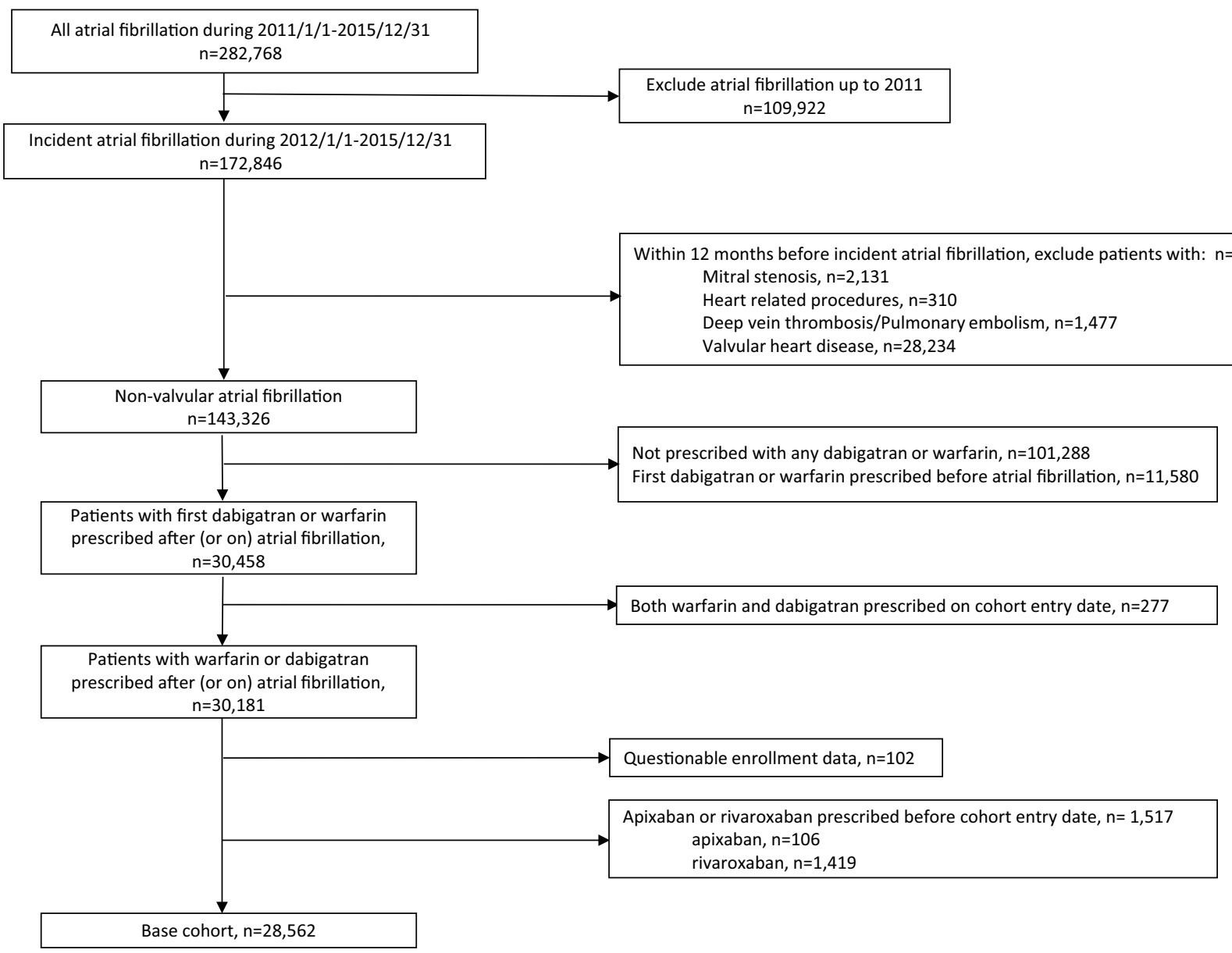

Fig. 1 Development of base cohort

Table 1 Date of approval for non-vitamin $\mathrm{K}$ oral anticoagulants in Europe and reimbursement in Taiwan

\begin{tabular}{|c|c|c|c|c|}
\hline \multirow{2}{*}{$\begin{array}{l}\text { Non-vitamin } \\
\text { K oral antico- } \\
\text { agulants }\end{array}$} & \multicolumn{3}{|c|}{ Market approval } & \multirow{2}{*}{$\begin{array}{l}\text { Reim- } \\
\text { bursement } \\
\text { NHIA }\end{array}$} \\
\hline & US FDA & EMA & TFDA & \\
\hline Dabigatran & 2010/10/19 & 2008/03/18 & 2011/07/13 & 2012/06/01 \\
\hline Rivaroxaban & 2011/11/04 & $2008 / 09 / 30$ & $2012 / 03 / 08$ & 2013/02/01 \\
\hline Apixaban & $2012 / 12 / 28$ & $2011 / 05 / 18$ & 2013/08/14 & $2014 / 06 / 01$ \\
\hline Edoxaban & 2015/08/01 & $2017 / 04 / 20$ & $2016 / 02 / 02$ & 2016/09/01 \\
\hline
\end{tabular}

US FDA US Food and Drug Administration, EMA European Medicine Agency, TFDA Taiwan Food and Drug Administration, NHIA National Health Insurance Administration

probability of a patient switching from warfarin prescription to dabigatran.

\subsection{PS Matching and Study Cohort}

One warfarin prescription was matched to the initial dabigatran prescription within the same exposure set according to the estimated PS. A caliper of 0.06 standard deviation of logit PS within each exposure set was used to define the range of estimated $\mathrm{PS}$ within which to select the warfarin prescription. Warfarin prescription within the caliper of the corresponding dabigatran prescription and having the nearest PS to the dabigatran prescription was selected. The matching process was carried out in chronological order of when the exposure sets were formed. Once the patient with a warfarin prescription was matched to an initial dabigatran prescription, other warfarin prescriptions of the same patient in other exposure sets would no longer be eligible for matching to the yet to be matched new dabigatran patients. A patient may be matched as a warfarin patient to a dabigatran patient and later initiated with dabigatran, which was described as a prevalent new user (switcher) who was being "reused" [21]. The selected warfarin prescription and the corresponding new dabigatran prescription were index prescriptions and the prescription date became the index date for individual patients. While prescription was the unit utilized in formation of exposure sets and PS modeling within the base cohort, the corresponding individuals became the 
Table 2 Terminology used to describe incident and prevalent use of a drug

\begin{tabular}{|c|c|c|}
\hline Used in this report & $\begin{array}{l}\text { Described in published } \\
\text { literature [reference] }\end{array}$ & Definition \\
\hline Initiator & $\begin{array}{l}\text { New user }[4,41,42] \\
\text { Naïve user [4] } \\
\text { Incident user [2, 42] } \\
\text { Initiator [28] }\end{array}$ & $\begin{array}{l}\text { New users of the drug regardless of prior use of other drugs for the same disease } \\
\text { condition }\end{array}$ \\
\hline Incident new user or new user & $\begin{array}{l}\text { New user }[4,21] \\
\text { Naïve user [4] } \\
\text { Incident user [21] } \\
\text { Incident new user [21] }\end{array}$ & New user of a drug without prior use of any drug for the same disease condition \\
\hline Prevalent new user or switcher & $\begin{array}{l}\text { New user [4] } \\
\text { Switcher [21] } \\
\text { Prevalent new user [21] }\end{array}$ & New user of a drug with prior use of other drug(s) for the same disease condition \\
\hline $\begin{array}{l}\text { Prevalent user (not considered in } \\
\text { this report) }\end{array}$ & $\begin{array}{l}\text { Prevalent user }[4,41,42] \\
\text { Non-naïve user [4] } \\
\text { Continuing user [2] } \\
\text { Current user [2] }\end{array}$ & Already using the drug at the start of study period \\
\hline
\end{tabular}

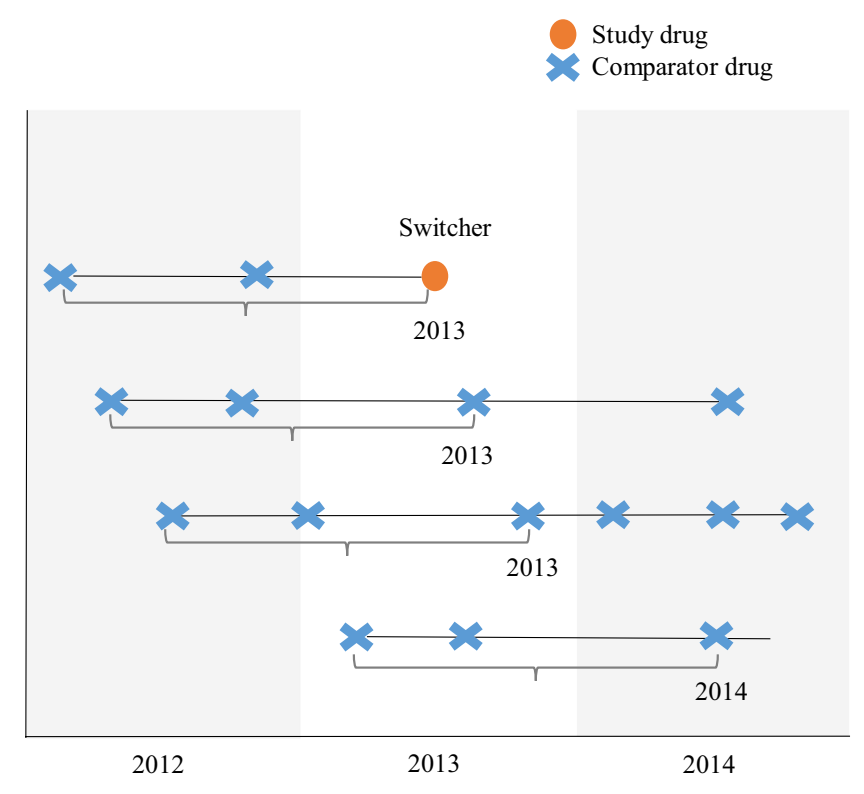

Fig. 2 Time- and prescription-based exposure set (matched on calendar year). For a comparator drug prescription to be included in an exposure set of a study drug, the comparator drug must have: (1) within 15-day difference of time from cohort entry, (2) the same

unit of analysis after 1-to-1 prescription matching. The study cohort comprised matched dabigatran and warfarin users, including both dabigatran incident and prevalent new users.

\subsection{Clinical Outcomes}

Outcomes of interest included intracranial hemorrhage, gastrointestinal bleeding, and ischemic stroke that resulted in hospital admission and all-cause mortality. Diagnosis codes that have been evaluated with Taiwan NHI data were used

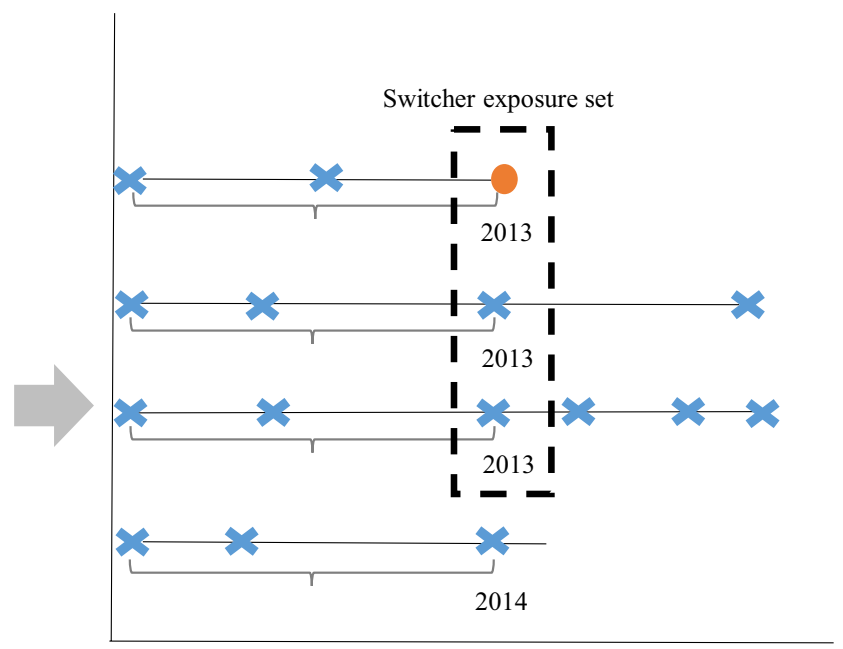

number of prior warfarin prescriptions, and (3) the same calendar year as the new dabigatran prescription is included in the exposure set of the new dabigatran prescription

[12]. Diagnosis code for ischemic stroke was validated with data from a single medical center in southern Taiwan, where $97.9 \%$ of $\mathrm{NHI}$ ischemic stroke cases were confirmed through review of medical records [24].

\subsection{Statistical Analysis}

One warfarin prescription representing a warfarin user of an exposure set was randomly sampled from each exposure set before PS matching to evaluate comparability between the 
dabigatran users and warfarin users. Clinical diagnoses and drug use 1 year prior to the dabigatran index prescriptions and the randomly selected warfarin index prescriptions were evaluated in order to assess the effect of balancing potential confounders between dabigatran and warfarin users with PS matching. Standardized mean differences for prevalence of covariates were calculated with a threshold of less than 0.1, implying well balanced characteristics [29].

Patients in the study cohort were followed from index date to the earliest of first event of interest, switching to another oral anti-coagulant, death, or 31 December 2015. Cox proportional hazard analysis was undertaken using the maximum partial likelihood method for estimation with a robust sandwich covariance matrix to account for the same patient contributing dabigatran-exposed person time and warfarin-exposed person time [30]. Hazard ratios (HRs) and the corresponding 95\% confidence intervals (CIs) were reported. Stratified analysis for incident and prevalent new users was performed to explore whether bleeding, ischemic stroke and mortality risks would be modified by prior warfarin use. All analyses were performed using SAS software, version 9.4 (SAS Institute, Inc, Cary, NC, USA).

\section{Results}

\subsection{Base Cohort}

We identified 143,326 incident NVAF patients in the Taiwan NHI from 2012 through 2015, with 30,458 patients having at least one warfarin or dabigatran prescription on or after the date of incident AF diagnosis. After excluding patients with incomplete NHI enrollment information, patients who received rivaroxaban or apixaban before receiving initiating dabigatran or warfarin, and those who were prescribed dabigatran and warfarin on the cohort entry date, the base cohort comprised 28,562 patients.

\subsection{Exposure Sets}

Among the base cohort AF patients, 528 were ineligible for evaluation in the prevalent new-user design due to having more than one oral anticoagulant prescribed on the same day after cohort entry. A total of 10,830 base cohort patients received dabigatran; $77 \%$ were incident new users and $23 \%$ were prevalent new users. As a result, 10,830 exposure sets could potentially be formed by an initial dabigatran prescription. After considering time and number of warfarin prescriptions from cohort entry up to the initial dabigatran prescriptions, 10,811 (99.8\%) initiating dabigatran prescriptions were able to find at least one eligible warfarin prescription to form time- and prescription-based exposure sets.

\subsection{Study Cohort after PS Matching}

After estimating PS for all dabigatran or warfarin prescriptions in each exposure set, a warfarin prescription having estimated PS being nearest within the caliper of that for the dabigatran prescription in the same exposure set was identified for 10,781 of dabigatran prevalent new users; 8385 (78\%) were dabigatran incident new users and 2396 (22\%) were prevalent new users.

Using one randomly selected warfarin user from each exposure set to evaluate the warfarin users' and dabigatran users' baseline characteristics, we found that the process of formulating the exposure sets according to prior warfarin use and time since first diagnosis of NVAF resulted in moderate comparability between the two groups (Supplementary Table 3). Dabigatran incident new users were older (mean age 75 years) than dabigatran prevalent new users (mean age 73 years) and had a lower prevalence of congestive heart failure, prior history of stroke, valvular heart disease, hemiplegia, and less concomitant use of statins (Supplementary Table 4). For the study cohorts after PS matching, mean age was 74 years and about $60 \%$ were male. Approximately $70 \%$ of patients had hypertension, $30 \%$ had diabetes, and $10 \%$ had renal impairment. Prevalence of all measured baseline covariates was balanced between the two study cohorts (Table 3).

\subsection{Clinical Outcomes}

Median duration of follow-up among the study cohort was 0.9 year. Incidence rates among dabigatran prevalent new users for intracranial hemorrhage, gastrointestinal bleeding, and ischemic stroke were $0.70,3.17$, and 4.91 per 100 person-year, respectively, and were $1.38,3.96$, and 5.44 per 100 person-year, respectively, among warfarin users. There were 1472 prevalent new users whose prior warfarin-exposed person time before initial dabigatran use was matched to another dabigatran user. The intracranial hemorrhage, gastrointestinal bleeding, and ischemic stroke incidence during the prior warfarin-exposed person time were 1.50, 3.67, and 11.48 per 100 person-year.

Decreased risk of intracranial hemorrhage $(\mathrm{HR}=0.51$; 95\% CI $0.39,0.66)$ and gastrointestinal bleeding $(\mathrm{HR}=0.81 ; 95 \% \mathrm{CI} 0.70,0.92)$ were observed among dabigatran initiators compared with the warfarin users (Table 4). There was no strong evidence to suggest a lower risk of ischemic stroke among dabigatran and warfarin users (HR $=0.91$; 95\% CI: 0.81, 1.02). Dabigatran initiators had a reduced risk for all-cause mortality $(\mathrm{HR}=0.59$; 95\% CI 0.54, 0.64) (Table 5).

In the subgroup analyses stratified by incident new users and prevalent new users, similar levels of lower risk of major bleeding with dabigatran use were observed compared with 
Table 3 Characteristics of study subjects, propensity score-matched dabigatran and warfarin users in time- and prescription-based exposure sets $(1$ year before index prescription)

\begin{tabular}{|c|c|c|c|c|c|}
\hline \multirow[b]{3}{*}{$n,(\%)$} & \multicolumn{5}{|c|}{ After propensity score match } \\
\hline & Dabigatran & & \multicolumn{2}{|l|}{ Warfarin } & \multirow[t]{2}{*}{$\begin{array}{l}\text { Standard- } \\
\text { ized differ- } \\
\text { ence }\end{array}$} \\
\hline & 10,781 & & 10,781 & & \\
\hline Mean age, year (SD) & 74.52 & 10.16 & 73.79 & 10.98 & 0.07 \\
\hline Male & 6329 & $59 \%$ & 6276 & $58 \%$ & 0.01 \\
\hline \multicolumn{6}{|l|}{$\mathrm{CHA}_{2} \mathrm{DS}_{2}$-VASc } \\
\hline Mean (SD) & 3.90 & 1.64 & 3.77 & 1.85 & 0.07 \\
\hline Median (Q1-Q3) & 4.00 & $(3-5)$ & 4.00 & $(2-5)$ & \\
\hline Congestive heart failure & 3209 & $30 \%$ & 3301 & $31 \%$ & -0.02 \\
\hline Hypertension & 7768 & $72 \%$ & 7674 & $71 \%$ & 0.02 \\
\hline Chronic pulmonary disease & 2308 & $21 \%$ & 2248 & $21 \%$ & 0.01 \\
\hline Diabetes & 3237 & $30 \%$ & 3255 & $30 \%$ & 0.00 \\
\hline Renal failure & 1170 & $11 \%$ & 1287 & $12 \%$ & -0.03 \\
\hline Liver disease & 920 & $9 \%$ & 924 & $9 \%$ & 0.00 \\
\hline Peptic ulcer disease excluding bleeding & 1260 & $12 \%$ & 1258 & $12 \%$ & 0.00 \\
\hline Ischemic stroke & 3229 & $30 \%$ & 3010 & $28 \%$ & 0.04 \\
\hline Intracranial hemorrhage & 210 & $2 \%$ & 209 & $2 \%$ & 0.00 \\
\hline Gastrointestinal bleeding & 584 & $5 \%$ & 612 & $6 \%$ & -0.01 \\
\hline Myocardial infarction & 350 & $3 \%$ & 383 & $4 \%$ & -0.02 \\
\hline Vascular disease & 706 & $7 \%$ & 735 & $7 \%$ & -0.01 \\
\hline Hemiplegia or paraplegia & 579 & $5 \%$ & 605 & $6 \%$ & -0.01 \\
\hline Dementia & 605 & $6 \%$ & 605 & $6 \%$ & 0.00 \\
\hline Gout & 1013 & $9 \%$ & 1066 & $10 \%$ & -0.02 \\
\hline Percutaneous coronary intervention & 215 & $2 \%$ & 228 & $2 \%$ & -0.01 \\
\hline Coronary artery bypass surgery & 7 & $0 \%$ & 25 & $0 \%$ & -0.04 \\
\hline Antiplatelet & 3271 & $30 \%$ & 3211 & $30 \%$ & 0.01 \\
\hline Low-dose aspirin & 7144 & $66 \%$ & 6923 & $64 \%$ & 0.04 \\
\hline NSAID & 7101 & $66 \%$ & 7188 & $67 \%$ & -0.02 \\
\hline Antiepileptics & 292 & $3 \%$ & 290 & $3 \%$ & 0.00 \\
\hline Antifungal & 130 & $1 \%$ & 126 & $1 \%$ & 0.00 \\
\hline Diuretics & 2677 & $25 \%$ & 2658 & $25 \%$ & 0.00 \\
\hline Beta-blockers & 6938 & $64 \%$ & 6979 & $65 \%$ & -0.01 \\
\hline CCB & 7341 & $68 \%$ & 7306 & $68 \%$ & 0.01 \\
\hline ACEI & 2369 & $22 \%$ & 2404 & $22 \%$ & -0.01 \\
\hline ARB & 6284 & $58 \%$ & 6164 & $57 \%$ & 0.02 \\
\hline Statin & 3812 & $35 \%$ & 3694 & $34 \%$ & 0.02 \\
\hline Other lipid-lowering agents & 680 & $6 \%$ & 693 & $6 \%$ & 0.00 \\
\hline Insulin & 1200 & $11 \%$ & 1271 & $12 \%$ & -0.02 \\
\hline Other oral antidiabetics & 2967 & $28 \%$ & 2970 & $28 \%$ & 0.00 \\
\hline Dabigatran new users & 8385 & $78 \%$ & & & \\
\hline Dabigatran switchers & 2396 & $22 \%$ & & & \\
\hline
\end{tabular}

$S D$ standard deviation, $Q 1-Q 3$ quartile 1-quartile 3, NSAID nonsteroidal anti-inflammatory drug, $C C B$ calcium channel blocker, $A C E I$ angiotensin-converting enzyme inhibitor, $A R B$ angiotensin receptor blocker warfarin. For gastrointestinal bleeding, HR was 0.84 for the dabigatran incident new users and it was 0.69 among the prevalent new users (Table 4). On the other hand, ischemic stroke risk was lower among incident new users of dabigatran $(\mathrm{HR}=0.84 ; 95 \% \mathrm{CI} 0.74,0.96)$, but not observed among prevalent new users $(\mathrm{HR}=1.19 ; 95 \% \mathrm{CI} 0.94,1.50)$. The reduction in all-cause mortality rate with dabigatran was similar in incident $(\mathrm{HR}=0.59)$ and prevalent new users $(\mathrm{HR}=0.57)$ when compared to warfarin users (Table 5). 
Table 4 Bleeding outcome analyses

\begin{tabular}{|c|c|c|c|c|c|}
\hline Outcomes & Total & $\begin{array}{l}\text { Number of } \\
\text { events }\end{array}$ & Total person-year & $\begin{array}{l}\text { Incidence rate (per } \\
100 \text {-person year) }\end{array}$ & $\begin{array}{l}\text { Matched hazard } \\
\text { ratio }(95 \% \mathrm{CI})\end{array}$ \\
\hline \multicolumn{6}{|c|}{ Intracranial hemorrhage } \\
\hline Dabigatran & 10,781 & 85 & $12,159.64$ & 0.70 & $0.51(0.39,0.66)$ \\
\hline Warfarin & 10,781 & 161 & $11,664.71$ & 1.38 & 1 \\
\hline \multicolumn{6}{|c|}{ Incident new users } \\
\hline Dabigatran & 8385 & 65 & 9304.59 & 0.70 & $0.50(0.37,0.68)$ \\
\hline Warfarin & 8385 & 122 & 8726.07 & 1.40 & 1 \\
\hline \multicolumn{6}{|c|}{ Prevalent new users } \\
\hline Dabigatran & 2396 & 20 & 2855.05 & 0.70 & $0.53(0.31,0.91)$ \\
\hline Warfarin & 2396 & 39 & 2938.63 & 1.33 & 1 \\
\hline \multicolumn{6}{|c|}{ Gastrointestinal bleeding } \\
\hline Dabigatran & 10,781 & 379 & $11,959.77$ & 3.17 & $0.81(0.70,0.92)$ \\
\hline Warfarin & 10,781 & 452 & $11,416.87$ & 3.96 & 1 \\
\hline \multicolumn{6}{|c|}{ Incident new users } \\
\hline Dabigatran & 8385 & 304 & 9136.81 & 3.33 & $0.84(0.72,0.98)$ \\
\hline Warfarin & 8385 & 341 & 8551.01 & 3.99 & 1 \\
\hline \multicolumn{6}{|c|}{ Prevalent new users } \\
\hline Dabigatran & 2396 & 75 & 2822.96 & 2.66 & $0.69(0.51,0.92)$ \\
\hline Warfarin & 2396 & 111 & 2865.86 & 3.87 & 1 \\
\hline
\end{tabular}

CI confidence interval
Table 5 Effectiveness outcome analyses

\begin{tabular}{|c|c|c|c|c|c|}
\hline Outcomes & Total & $\begin{array}{l}\text { Number of } \\
\text { events }\end{array}$ & Total person-year & $\begin{array}{l}\text { Incidence rate (per } \\
100 \text {-person year) }\end{array}$ & $\begin{array}{l}\text { Matched hazard } \\
\text { ratio }(95 \% \mathrm{CI})\end{array}$ \\
\hline \multicolumn{6}{|c|}{ Ischemic stroke } \\
\hline Dabigatran & 10,781 & 580 & $11,820.41$ & 4.91 & $0.91(0.81,1.02)$ \\
\hline Warfarin & 10,781 & 614 & $11,297.11$ & 5.44 & 1 \\
\hline \multicolumn{6}{|c|}{ Incident new users } \\
\hline Dabigatran & 8385 & 438 & 9055.96 & 4.84 & $0.84(0.74,0.96)$ \\
\hline Warfarin & 8385 & 490 & 8437.16 & 5.81 & 1 \\
\hline \multicolumn{6}{|c|}{ Prevalent new users } \\
\hline Dabigatran & 2396 & 142 & 2764.45 & 5.14 & $1.19(0.94,1.50)$ \\
\hline Warfarin & 2396 & 124 & 2859.95 & 4.34 & 1 \\
\hline \multicolumn{6}{|c|}{ All-cause mortality } \\
\hline Dabigatran & 10,781 & 872 & $12,214.31$ & 7.14 & $0.59(0.54,0.64)$ \\
\hline Warfarin & 10,781 & 1437 & $11,748.9$ & 12.23 & 1 \\
\hline \multicolumn{6}{|c|}{ Incident new users } \\
\hline Dabigatran & 8385 & 707 & 9344.38 & 7.57 & $0.59(0.53,0.65)$ \\
\hline Warfarin & 8385 & 1138 & 8793.46 & 12.94 & 1 \\
\hline \multicolumn{6}{|c|}{ Prevalent new users } \\
\hline Dabigatran & 2396 & 165 & 2869.93 & 5.75 & $0.57(0.47,0.69)$ \\
\hline Warfarin & 2396 & 299 & 2955.44 & 10.12 & 1 \\
\hline
\end{tabular}

CI confidence interval

\section{Discussion}

In this population-based study conducted within an incident NVAF base cohort, we identified 8385 dabigatran incident new users (new users) and 2396 prevalent new users (switchers) in a nationwide data environment and implemented the prevalent new-user design to evaluate comparative safety and effectiveness of dabigatran and warfarin. The novel design allows for studying incident new users and prevalent new users in the same analysis through time- and 
prescription-based exposure sets. Comparable warfarin users were identified for both dabigatran incident and prevalent new users, and the dabigatran users had a significant reduction in intracranial hemorrhage and gastrointestinal bleeding risks. Dabigatran was more effective in the prevention of ischemic stroke among the incident new users but the same effect was not observed among the prevalent new users.

Hernan and Robins proposed the "target trial" concept, and within that framework [31], the prevalent new-user design would emulate a trial that allows for patient enrollment regardless of prior use of the comparator drug. Within a pool of eligible subjects with the same usage experience of the comparator drug with regard to the number of prescriptions within a defined baseline timeframe, one subject would be randomly assigned to the new drug and another would be randomly assigned to the comparator.

If dabigatran is evaluated in a conventional new-user design without considering prior warfarin use $[1,4]$, the nearly 10,000 dabigatran initiators in Taiwan would form a heterogeneous group, introducing potential confounding. One the other hand, if the study population only comprised dabigatran initiators who did not use warfarin previously, study size would be reduced by $22 \%$ and there would be no information on the prevalent new users (switchers). With the use of prevalent new user design in the post-marketing assessment of dabigatran in a healthcare system, virtually all incident NVAF patients prescribed dabigatran regardless of prior warfarin use were evaluated in the same analysis, providing comprehensive safety experience of dabigatran use as well as the capacity to evaluate drug effects separately among incident and prevalent new users. The design is most relevant in a therapeutic area where the safety of a novel medication is assessed in comparison with an existing regimen.

Our findings on intracranial hemorrhage, gastrointestinal bleeding, ischemic stroke, and all-cause mortality in the dabigatran versus warfarin comparison were within the range of those reported from prior studies from Denmark and the USA [8, 10, 11, 27, 32]. The longitudinal nature of the Taiwan NHI allows for extended look-back period to evaluate warfarin use before dabigatran initiation, which may not be feasible in health insurance claims data in the USA. In the US studies, look-back periods ranged from 3 months to 12 months [8, 16, 33], and may result in misclassification of prior warfarin use and limited ascertainment of prior warfarin use duration. The longitudinal nature of the Taiwan NHI is more similar to the Danish data environment, where ascertainment of prior warfarin use may be comprehensively addressed either by the design of exposure sets or other analytical methods. In a Danish study that evaluated bleeding risks among NOACs and warfarin users, prior warfarin use experience was evaluated as a covariate in multiple regression models [13]. In applying the prevalent new user design, the use of exposure sets for index prescriptions in the same calendar year could simultaneously control for the duration and frequency of prior warfarin use in a prospective manner, which offered a transparent alternative to conventional cohort design with time-dependent analyses in a Cox model. A similar approach could be applied for the evaluation of rivaroxaban, another NOAC [17]. However, the realworld drug use scenario is more complex for subsequently approved NOACs, such as apixaban and edoxaban, as there would be switching from warfarin and between NOACs. The prevalent new user design is also applicable in head-to head comparisons amongst different NOACS [33-36]. However, the design of exposure sets and PS estimation would be more complicated, as the design needs to take into account for various patterns of prior warfarin and NOACs use. Furthermore, the implementation of prevalent new user design would be more complicated in disease conditions for which a drug of interest may be used in monotherapy and add-on therapy. For NVAF only monotherapy with an oral anticoagulant is clinically indicated, but that is not the case for other diseases. For instance, long-acting inhaled corticosteroids may be added to a beta-adrenergic agonist for combination treatment for chronic obstructive pulmonary disease [37] or a sulfonylurea as a second-line oral antidiabetic agent would be added to first-line metformin in diabetic patients [22]. When a new drug of interest is added on to the existing regimen, defining additional exposure group is suggested to complement the incident and prevalent new users for cohort studies [22].

Findings from this study were mostly consistent with those from prior reports from Taiwan using the same nationwide data over different time periods [10, 11, 38]. Dabigatran use was associated with approximately $50 \%$ reduced risk of intracranial hemorrhage and all-cause mortality $[10,11]$. The $20 \%$ risk reduction in gastrointestinal bleeding observed in our study is similar to that of another report (HR $=0.77 ; 95 \%$ CI 0.59, 1.02) [38], although two earlier studies reported a similar gastrointestinal bleeding risk among dabigatran and warfarin users [10,11]. As for ischemic stroke, we did not find a favorable ischemic stroke prevention effect for dabigatran, and previous studies from Taiwan reported $20-40 \%$ ischemic stroke risk reduction associated with dabigatran $[10,11,38]$. A methodology concern for prior studies was that for recurrent events such as bleeding or ischemic stroke, event rates during warfarinexposed person-time before dabigatran initiation among prevalent new users were not ascertained in conventional retrospective cohort studies [11]. Studying prevalent new users without addressing comparable exposed person-time and not ascertaining outcome events during such persontime may introduce bias in prevalent user designs [4]. In this study, rates of outcomes of interest during the pre-dabigatran warfarin-exposed person-time were ascertained and major 
bleeding rate was similar to that during overall warfarinexposed person time and ischemic stroke incidence rate was higher during the prior warfarin use person-time. These findings suggested under-ascertainment of early events in the prior warfarin use among dabigatran prevalent new users in previous study [4].

With the use of time- and prescription-based exposure sets in prevalent new user design, we found that distributions of potential confounders, except for renal failure and age, between dabigatran and warfarin users were empirically balanced before PS matching, illustrating an efficient means to control for potential confounders without using complex statistical analysis. After matching on time-conditional PS, distribution of all measured confounders was balanced between dabigatran and warfarin users. In this study with prevalent new-user design, given that comparable warfarin users were selected within the exposure sets and the subgroup of interest was prior warfarin use status, subgroup analysis of incident and prevalent new user could be performed without breaking the matched sets, while retaining balanced characteristics from PS matching. A methodology review suggested that most propensity matched studies (33\% of the 83 studies reviewed) appeared to perform subgroup analysis according to any variable of interest directly from the matched cohorts, which would break the PS-matched sets and patient characteristics may no longer be balanced within the subgroups [39]. We suspect that similar issues may also be present in subgroup analysis along with inverse probability of treatment weighting where the cohort was subdivided directly from the weighted cohort. Although a recent simulation study suggested that limited bias was observed for breaking matched sets in subgroup analysis, the authors concluded that it was due to the low prevalence of broken matches. If more covariates were added to the PS model or high dimensional PS models were used, prevalence of broken matches may not be low in subgroup analysis [40]. In the context of prevalent new-user design studies, incident or prevalent new-user status were of primary interest and thus evaluation of these subgroups would be very straightforward. However, subgroup analyses on variables other than prior active comparator use would face similar concerns on imbalanced confounder distribution after stratification.

While the public health insurance program in Taiwan generated extensive longitudinal data with minimal loss to follow-up, our limited study budget only allowed for access to NHI data starting from 2011. As such, dabigatran users for whom AF onset was before 2011 were not included in the study. It did not affect the validity of the inference, but the number of dabigatran prevalent new users could be increased if an earlier data access time was available. Patients who had AF onset before 2011, received warfarin, and subsequently switched to dabigatran would be identified, resulting in a larger number of dabigatran prevalent new users in the study. As in any study based on health insurance claims, lack of lifestyle factors and laboratory examination results, especially international normalized ratio (INR) for monitoring warfarin use, are the limitations. Although INR tests are required for warfarin dose adjustment, when both frequency and duration of prior warfarin treatment are accounted in the exposure sets, the warfarin utilization information captured in the exposure sets may indirectly control for anticoagulant treatment effects and mitigate the limitations of no INR data in the study.

\section{Conclusion}

In conclusion, we utilized a novel design that took into account prior drug-use history in post-marketing safety evaluation of a new drug (dabigatran) after it was introduced to Taiwan. The method could be applied to the evaluation of all new drugs in similar post-marketing settings.

Acknowledgements Data in this study were provided by the Health and Welfare Data Science Center (HWDSC) of the Department of Statistics, Ministry of Health and Welfare, Taipei, Taiwan.

\section{Compliance with Ethical Standards}

Ethics Review The study was approved by the Institutional Review Board of the National Taiwan University Hospital Hsin-Chu Branch (approval document 104-009-E) and individual authorization by study subjects was waived for the use of anonymous data.

Conflict of interest $\mathrm{K}$. Arnold Chan is the principal investigator for several industry-sponsored studies and Hui-Min Diana Lin is employed through one of the research contracts, none of them are related to this report. Chao-Lun Lai reports receiving lecture fees from AstraZeneca, Pfizer, Bayer AG, Novartis, Actelion, Boehringer Ingelheim, Excelsior, Sanofi-Aventis, MSD, Tanabe, Daiichi-Sankyo, and Abbott. Samy Suissa has received research funding, participated in advisory board meetings or as speaker for Bayer, Boehringer-Ingelheim, and Bristol-Myers-Squibb. Yaa-Hui Dong and Yu-Kang Tu have no conflicts of interest to disclose.

Funding This work was supported by Grants from the National Taiwan University Hospital, Hsin-Chu Branch (105-HCH016 and 105$\mathrm{HCH003).}$

Open Access This article is distributed under the terms of the Creative Commons Attribution-NonCommercial 4.0 International License (http://creativecommons.org/licenses/by-nc/4.0/), which permits any noncommercial use, distribution, and reproduction in any medium, provided you give appropriate credit to the original author(s) and the source, provide a link to the Creative Commons license, and indicate if changes were made. 


\section{References}

1. Lund JL, Richardson DB, Stürmer T. The active comparator, new user study design in pharmacoepidemiology: historical foundations and contemporary application. Curr Epidemiol Rep. 2015;2:221-8.

2. Johnson ES, Bartman BA, Briesacher BA, Fleming NS, Gerhard T, Kornegay CJ, et al. The incident user design in comparative effectiveness research. Pharmacoepidemiol Drug Saf. 2013;22:1-6.

3. Hernan MA, Alonso A, Logan R, Grodstein F, Michels KB, Willett WC, et al. Observational studies analyzed like randomized experiments: an application to postmenopausal hormone therapy and coronary heart disease. Epidemiology. 2008;19:766-79.

4. Ray WA. Evaluating medication effects outside of clinical trials: new-user designs. Am J Epidemiol. 2003;158:915-20.

5. Toh S, Reichman ME, Houstoun M, Ross Southworth M, Ding X, Hernandez AF, et al. Comparative risk for angioedema associated with the use of drugs that target the renin-angiotensin-aldosterone system. Arch Intern Med. 2012;172:1582-9.

6. Larsen TB, Rasmussen LH, Skjøth F, Due KM, Callréus T, Rosenzweig M, et al. Efficacy and safety of dabigatran etexilate and warfarin in "real-world" patients with atrial fibrillation: a prospective nationwide cohort study. J Am Coll Cardiol. 2013;61:2264-73.

7. Bengtson L, Lutsey P, Chen L, MacLehose R, Alonso A. Comparative effectiveness of dabigatran and rivaroxaban versus warfarin for the treatment of non-valvular atrial fibrillation. J Cardiol. 2017;69(6):868-76.

8. Graham DJ, Reichman ME, Wernecke M, Zhang R, Southworth MR, Levenson M, et al. Cardiovascular, bleeding, and mortality risks in elderly Medicare patients treated with dabigatran or warfarin for nonvalvular atrial fibrillation. Circulation. 2015;131:157-64.

9. Lauffenburger JC, Farley JF, Gehi AK, Rhoney DH, Brookhart MA, Fang G. Effectiveness and safety of dabigatran and warfarin in real-world US patients with non-valvular atrial fibrillation: a retrospective cohort study. J Am Heart Assoc. 2015;4:1-12.

10. Chan YH, Kuo CT, Yeh YH, Chang SH, Wu LS, Lee HF, et al. Thromboembolic, bleeding, and mortality risks of rivaroxaban and dabigatran in asians with nonvalvular atrial fibrillation. J Am Coll Cardiol. 2016;68:1389-401.

11. Chan YH, Yen KC, See LC, Chang SH, Wu LS, Lee HF, et al. Cardiovascular, bleeding, and mortality risks of dabigatran in asians with nonvalvular atrial fibrillation. Stroke. 2016;47:441-9.

12. Lai CL, Chen HM, Liao MT, Lin TT, Chan KA. Comparative effectiveness and safety of dabigatran and rivaroxaban in atrial fibrillation patients. J Am Heart Assoc. 2017;6(4):e005362.

13. Larsen TB, Gorst-Rasmussen A, Rasmussen LH, Skjøth F, Rosenzweig M, Lip GYH. Bleeding events among new starters and switchers to dabigatran compared with warfarin in atrial fibrillation. Am J Med. 2014;127:650-6.

14. Sørensen R, Gislason G, Torp-Pedersen C, Olesen JB, Fosbøl EL, Hvidtfeldt MW, et al. Dabigatran use in Danish atrial fibrillation patients in 2011: a nationwide study. BMJ Open. 2013;3:e002758.

15. Staerk L, Gislason GH, Lip GY, Fosbol EL, Hansen ML, Lamberts M, et al. Risk of gastrointestinal adverse effects of dabigatran compared with warfarin among patients with atrial fibrillation: a nationwide cohort study. Europace. 2015;17:1215-22.

16. Seeger JD, Bykov K, Bartels DB, Huybrechts K, Zint K, Schneeweiss S. Safety and effectiveness of dabigatran and warfarin in routine care of patients with atrial fibrillation. Thromb Haemost. 2015; 114:1277-89.

17. Chrischilles EA, Gagne JJ, Fireman B, Nelson J, Toh S, Shoaibi A, et al. Prospective surveillance pilot of rivaroxaban safety within the US Food and Drug Administration Sentinel System. Pharmacoepidemiol Drug Saf. 2018;27:263-71.

18. Southworth MR, Reichman ME, Unger EF. Dabigatran and postmarketing reports of bleeding. N Engl J Med. 2013;368:1272-4.

19. Moride Y, Abenhaim L. Evidence of the depletion of susceptibles effect in non-experimental pharmacoepidemiologic research. J Clin Epidemiol. 1994;47:731-7.

20. McMahon AD, MacDonald TM. Design issues for drug epidemiology. Br J Clin Pharmacol. 2000;50:419-25.

21. Suissa S, Moodie EE, Dell'Aniello S. Prevalent new-user cohort designs for comparative drug effect studies by timeconditional propensity scores. Pharmacoepidemiol Drug Saf. 2017;26:459-68.

22. Douros A, Dell'Aniello S, Yu OHY, Filion KB, Azoulay L, Suissa $\mathrm{S}$. Sulfonylureas as second line drugs in type 2 diabetes and the risk of cardiovascular and hypoglycaemic events: population based cohort study. BMJ. 2018;362:k2693.

23. Hsiao FY, Yang CL, Huang YT, Huang WF. Using Taiwan's national health insurance research databases for pharmacoepidemiology research. J Food Drug Anal. 2007;15:99-108.

24. Cheng CL, Kao YH, Lin SJ, Lee CH, Lai ML. Validation of the National Health Insurance Research Database with ischemic stroke cases in Taiwan. Pharmacoepidemiol Drug Saf. 2011;20:236-42.

25. Cheng CL, Lee CH, Chen PS, Li YH, Lin SJ, Yang YHK. Validation of acute myocardial infarction cases in the national health insurance research database in Taiwan. J Epidemiol. 2014;24:500-7.

26. Lu TH, Lee MC, Chou MC. Accuracy of cause-of-death coding in Taiwan: types of miscoding and effects on mortality statistics. Int J Epidemiol. 2000;29:336-43.

27. Larsen TB, Rasmussen LH, Gorst-Rasmussen A, Skjøth F, Lane DA, Lip GYH. Dabigatran and warfarin for secondary prevention of stroke in atrial fibrillation patients: a nationwide cohort study. Am J Med. 2014;127(1172-8):e5.

28. Funch D, Gydesen H, Tornøe K, Major-Pedersen A, Chan KA. A prospective, claims-based assessment of the risk of pancreatitis and pancreatic cancer with liraglutide compared to other antidiabetic drugs. Diabetes Obes Metab. 2014;16:273-5.

29. Austin P. An introduction to propensity score methods for reducing the effects of confounding in observational studies. Multivariate Behav Res. 2011;46:399-424.

30. Lee EW, Wei LJ, Amato DA, Leurgans S. Cox-type regression analysis for large numbers of small groups of correlated failure time observations. In: Klein JP, Goel PK, editors. Survival analysis: state of the art. Dordrecht: Springer; 1992. p. 237-47.

31. Hernán M, Robins J. Using big data to emulate a target trial when a randomized trial is not available. Am J Epidemiol. 2016;183(8):758-64.

32. Yao X, Abraham NS, Sangaralingham LR, Bellolio MF, McBane $\mathrm{RD}$, Shah ND, et al. Effectiveness and safety of dabigatran, rivaroxaban, and apixaban versus warfarin in nonvalvular atrial fibrillation. J Am Heart Assoc. 2016;5(6):e003725.

33. Hernandez I, Zhang Y. Comparing stroke and bleeding with rivaroxaban and dabigatran in atrial fibrillation: analysis of the US Medicare part D data. Am J Cardiovasc Drugs. 2017;17:37-47.

34. Graham DJ, Reichman ME, Wernecke M, Hsueh YH, Izem R, Southworth MR, et al. Stroke, bleeding, and mortality risks in elderly medicare beneficiaries treated with dabigatran or rivaroxaban for nonvalvular atrial fibrillation. JAMA Intern Med. 2016;176:1662-71.

35. Gorst-Rasmussen A, Lip GY, Larsen Bjerregaard T. Rivaroxaban versus warfarin and dabigatran in atrial fibrillation: comparative effectiveness and safety in Danish routine care. Pharmacoepidemiol Drug Saf. 2016;25:1236-44. 
36. Noseworthy PA, Yao X, Abraham NS, Sangaralingham LR, McBane RD, Shah ND. Direct comparison of dabigatran, rivaroxaban, and apixaban for effectiveness and safety in nonvalvular atrial fibrillation. Chest. 2016;150:1302-12.

37. Dong YH, Chang CH, Gagne JJ, Hsu CL, Lai MS. Comparative cardiovascular and cerebrovascular safety of inhaled long-acting bronchodilators in patients with chronic obstructive pulmonary disease: a population-based cohort study. Pharmacotherapy. 2016;36:26-37.

38. Chan YH, See LC, Tu HT, Yeh YH, Chang SH, Wu LS, et al. Efficacy and safety of apixaban, dabigatran, rivaroxaban, and warfarin in asians with nonvalvular atrial fibrillation. J Am Heart Assoc. 2018;7(8):e008150.

39. Wang SV, He M, Jin Y, Wyss R, Shin H, Ma Y, et al. A review of the performance of different methods for propensity score matched subgroup analyses and a summary of their application in peer-reviewed research studies. Pharmacoepidemiol Drug Saf. 2017;26:1507-12.

40. Wang SV, Jin Y, Fireman B, Gruber S, He M, Wyss R, et al. Relative performance of propensity score matching strategies for subgroup analyses. Am J Epidemiol. 2018;187:1799-807.

41. Gokhale M, Buse JB, Pate V, Marquis MA, Sturmer T. More realistic power estimation for new user, active comparator studies: an empirical example. Pharmacoepidemiol Drug Saf. 2016;25:462-6.

42. Vandenbroucke J, Pearce N. Point: incident exposures, prevalent exposures, and causal inference: does limiting studies to persons who are followed from first exposure onward damage epidemiology? Am J Epidemiol. 2015;182:826-33.

\section{Affiliations}

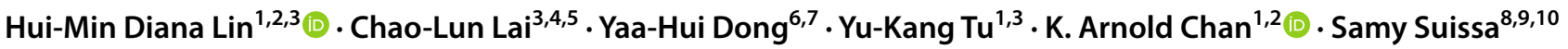

1 Department of Medical Research, National Taiwan University Hospital, No.7, Chung Shan South Road, Taipei, Taiwan

2 Health Data Research Center, National Taiwan University, No.33, Linsen South Road, Suite 526, Taipei, Taiwan

3 Institute of Epidemiology and Preventive Medicine, College of Public Health, National Taiwan University, Room 501, No. 17, Xu-Zhou Road, Taipei, Taiwan

4 Department of Internal Medicine, National Taiwan University Hospital, Hsin-Chu Branch, No.25, Lane 442, Section 1, Jingguo Road, Hsinchu, Taiwan

5 Department of Internal Medicine, College of Medicine, National Taiwan University, No.1, Section 1, Jen Ai Road, Taipei, Taiwan
6 Faculty of Pharmacy, National Yang-Ming University, No.155, Section 2, Linong Street, Taipei, Taiwan

7 Institute of Public Health, National Yang-Ming University, No.155, Section 2, Linong Street, Taipei, Taiwan

8 Department of Epidemiology, Biostatistics, and Occupational Health, McGill University, Purvis Hall, 1020 Pine Avenue West, Montreal, Canada

$9 \quad$ McGill Pharmacoepidemiology Research Unit, McGill University, Purvis Hall, 1020 Pine Avenue West, Montreal, Canada

10 Centre for Clinical Epidemiology, Jewish General Hospital, 3755 Côte-Ste-Catherine Road, Montreal, Canada 